\section{Сопротивление и энергия при деформации почвы}

\author{
А. М. Цыпук ${ }^{1}$ \\ Петрозаводский государственный университет
}

\section{АННОТАЦИЯ}

Статья посвящена определению сил и затрат энергии, возникающих при вертикальном внедрении в почву лункообразователей различной формы. Использование методики рекомендуется при проектировании машин для точечной посадки леса. Приведены примеры расчетов.

Ключевые слова: лункообразователь, форма, почва, внедрение, сопротивление, энергия.

\section{SUMMARY}

The article is devoted to definition of forces and expenses of energy arising at vertical introduction in ground of the dibblers of various forms. Use of the technique is recommended at designing of the dibbling machines for planting trees. The examples of accounts are given.

Keywords: dibbler, form, ground, introduction, forces, energy.

При разработке машин для точечной посадки леса необходимо рассчитывать сопротивление внедрения в почву лункообразователей. При динамическом внедрении определяются также затраты энергии.

Наибольшее распространение получили лункообразователи пирамидальной, конической, клиновидной и секторной форм.

\section{СОПРОТИВЛЕНИЕ ВНЕДРЕНИЮ В ПОЧВУ ЛУНКООБРАЗОВАТЕЛЯ ПИРАМИДАЛЬНОЙ ФОРМЫ}

Рассмотрим методику определения силы $N$, необходимой для внедрения в почву лункообразователя, на примере четырехугольной усеченной пирамиды высотой $h$ (рис. 1).

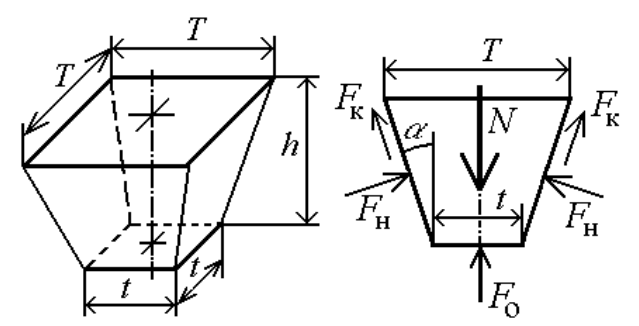

Рис. 1. Лункообразователь пирамидальной

\footnotetext{
${ }^{1}$ Автор - профессор кафедры технологии и оборудования лесного комплекса

(C) А. М. Цыпук, 2003
}

\section{формы}

Верхнее и нижнее основания пирамиды представляют собой квадраты со сторонами длиной соответственно $T$ и $t ; \alpha-$ угол наклона боковой грани пирамиды к вертикальной оси.

Из уравнения равновесия всех сил, приведенных к вертикальной оси пирамиды, получим выражение для искомой величины $N$ в общем виде:

$N=F_{\mathrm{o}}+4\left(F_{\mathrm{H}} \cdot \sin \alpha+F_{\mathrm{\kappa}} \cdot \cos \alpha\right)$.

Соотношение величин силы трения $F_{\text {К }}$ (касательной) и силы $F_{\text {H }}$ - нормальной (перпендикулярной к поверхности) определяется известным выражением:

$F_{\mathrm{K}}=F_{\mathrm{H}} \cdot f_{\mathrm{c}}$,

где $f_{\mathrm{c}}-$ коэффициент трения сталь-почва.

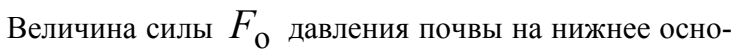
вание определится так:

$$
F_{\mathrm{o}}=S_{\mathrm{H}} \cdot[\sigma] \text {, }
$$

где $S_{\mathrm{H}}$ - площадь нижнего основания; $[\sigma]$ - удельное сопротивление почвы смятию.

Величина $F_{\mathrm{H}}$ определяется так:

$F_{\mathrm{H}}=S_{б} \cdot[\sigma]$,

где $S_{6}$ - площадь боковой грани пирамиды:

$S_{6}=\frac{h \cdot(T+t)}{2 \cos \alpha}$.

Подставляя выражения (2) - (5) в (1), получим:

$$
N=t^{2} \cdot[\sigma]+4 \cdot\left[\begin{array}{l}
\frac{h \cdot(T+t) \cdot[\sigma]}{2} \cdot \operatorname{tg} \alpha+ \\
+\frac{h \cdot(T+t) \cdot[\sigma] \cdot f_{\mathrm{c}}}{2}
\end{array}\right] .
$$

Выразим величину угла $\alpha$ через линейные параметры пирамиды (см. рис. 1):

$\operatorname{tg} \alpha=\frac{T-t}{2 h}$.

Подставим полученную величину в выражение (6). После сокращений получим:

$$
N=[\sigma] \cdot\left[T^{2}+2 h \cdot f_{\mathrm{c}} \cdot(T+t)\right] \text {. }
$$

Пример 1. Определить сопротивление внедрению в почву лункообразователя в виде усеченной четырехгранной пирамиды высотой 0,25 м, с размерами верхнего и нижнего основания соответственно $0,2 \times 0,2$ и $0,15 \times 0,15$ м. Величина удельного сопротивления 
почвы смятию 280 кПа, коэффициент трения стальпочва 0,26. Решение:

$N=280 \cdot 10^{3}\left[\begin{array}{l}0,2^{2}+2 \cdot 0,25 \cdot \\ \cdot 0,26 \cdot(0,2+0,15)\end{array}\right]=23940 \mathrm{H}$

Ответ: 23,94 кН.

\section{СОПРОТИВЛЕНИЕ ВНЕДРЕНИЮ В ПОЧВУ ЛУНКООБРАЗОВАТЕЛЯ КОНИЧЕСКОЙ ФОРМЫ}

Применим рассмотренную выше методику, основанную на использовании выражения (1), для определения силы $N_{1}$, необходимой для внедрения в почву лункообразователя в виде усеченного конуса, у которого радиусы верхнего и нижнего оснований соответственно $R$ и $r$ (рис. 2).

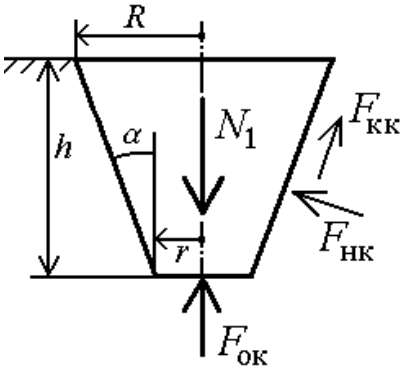

Рис. 2. Лункообразователь конической формы

Выражение для определения силы $N_{1}$ запишется так:

$N_{1}=F_{\text {ок }}+F_{\text {нк }} \cdot \sin \alpha+F_{\text {кк }} \cdot \cos \alpha$,

где $F_{\text {ок }}$ - сила давления почвы на нижнее основание усеченного конуса; $F_{\text {нк}}$ - суммарная сила давления почвы на боковую поверхность; $F_{\text {кК }}-$ суммарная сила трения почвы по боковой поверхности усеченного конуса.

Площадь $S_{\text {К }}$ боковой поверхности усеченного конуса может быть определена так:

$S_{\mathrm{\kappa}}=\frac{\pi \cdot\left(R^{2}-r^{2}\right)}{\sin \alpha}$.

В развернутом виде выражение (8) запишется так:

$$
\begin{aligned}
& N_{1}=\pi \cdot[\sigma] \cdot r^{2}+\frac{\pi \cdot[\sigma] \cdot\left(R^{2}-r^{2}\right)}{\sin \alpha} \cdot \sin \alpha+ \\
& +\frac{\pi \cdot[\sigma] \cdot\left(R^{2}-r^{2}\right)}{\sin \alpha} \cdot f_{\mathrm{c}} \cdot \cos \alpha= \\
& =\pi \cdot[\sigma] \cdot\left(R^{2}+\frac{R^{2}-r^{2}}{\operatorname{tg} \alpha} \cdot f_{\mathrm{c}}\right) .
\end{aligned}
$$

Выразим угловые параметры конуса через линейные: $\operatorname{tg} \alpha=\frac{R-r}{h}$.

В окончательном виде выражение для $N_{1}$ запишется так:

$$
N_{1}=\pi \cdot[\sigma] \cdot\left[R^{2}+f_{\mathrm{c}} \cdot h \cdot(R+r)\right] .
$$

\section{СОПРОТИВЛЕНИЕ ВНЕДРЕНИЮ В ПОЧВУ ЛУНКООБРАЗОВАТЕЛЯ КЛИНОВИДНОЙ ФОРМЫ}

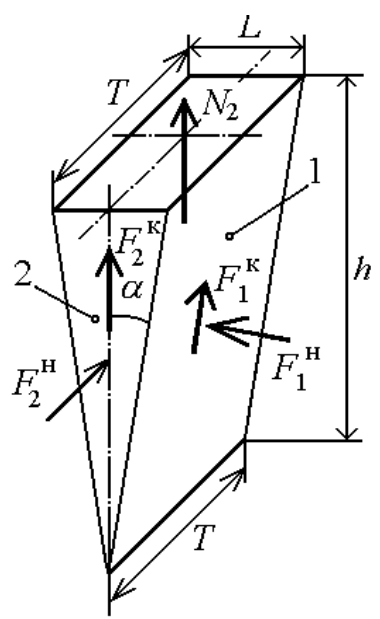

Рис. 3. Лункообразователь клиновидной формы

Используем рассмотренную выше методику, основанную на использовании выражения (1) для определения силы $N_{2}$, необходимой для внедрения в почву симметричного клина, фронтальные грани 1 у которого расположены под углом $\alpha$ к оси симметрии, а боковые грани 2 параллельны друг другу (см. рис. 3).

$N_{2}=2 F_{1}^{\mathrm{K}} \cdot \cos \alpha+2 F_{2}^{\mathrm{K}}+2 F_{1}^{\mathrm{H}} \cdot \sin \alpha$.

Здесь $F_{1}^{\mathrm{K}}$ - сила трения почвы по фронтальной грани; $F_{2}^{\mathrm{K}}$ - сила трения почвы по боковой грани; $F_{1}^{\mathrm{H}}-$ сила давления почвы на фронтальную грань.

Запишем выражения для составляющих (12):

$$
\begin{aligned}
& F_{1}^{\mathrm{K}}=F_{1}^{\mathrm{H}} \cdot f_{\mathrm{c}}=\frac{T \cdot h}{\cos \alpha} \cdot[\sigma] \cdot f_{\mathrm{c}} ; \\
& F_{1}^{\mathrm{H}}=\frac{T \cdot h}{\cos \alpha} \cdot[\sigma] ; \\
& F_{2}^{\mathrm{K}}=F_{2}^{\mathrm{H}} \cdot f_{\mathrm{c}}=\frac{L \cdot h}{2} \cdot[\sigma] \cdot f_{\mathrm{c}} .
\end{aligned}
$$

Здесь $F_{2}^{\mathrm{H}}$ - сила давления почвы на одну из боковых граней клина.

Перепишем (12) в развернутом виде: 
$\bar{N}_{2}=2 T \cdot h \cdot[\sigma] \cdot f_{\mathrm{c}}+L \cdot h \cdot[\sigma] \cdot f_{\mathrm{c}}+$ $+2 T \cdot h \cdot[\sigma] \cdot \operatorname{tg} \alpha$.

Выразим угол $\alpha$ через линейные параметры клина:

$\operatorname{tg} \alpha=\frac{L}{2 h}$.

В окончательном виде выражение для $N_{2}$ запишется так:

$$
N_{2}=[\sigma] \cdot\left[T \cdot L+(2 T+L) \cdot h \cdot f_{\mathrm{c}}\right] \text {. }
$$

СОПРОТИВЛЕНИЕ ВНЕДРЕНИЮ В ПОЧВУ ЛУНКООБРАЗОВАТЕЛЯ СЕКТОРНОЙ ФОРМЫ Примем допущение, что величина силы $N_{3}$, необходимой для внедрения сектора в виде четверти круга радиусом $R$ и толщиной $T$ (см. рис. 4) на глубину $h$, составит половину величины силы, необходимой для вдавливания полукруга, плюс сила трения по боковой грани сектора, вызванная упруго-пластичной деформацией почвы.

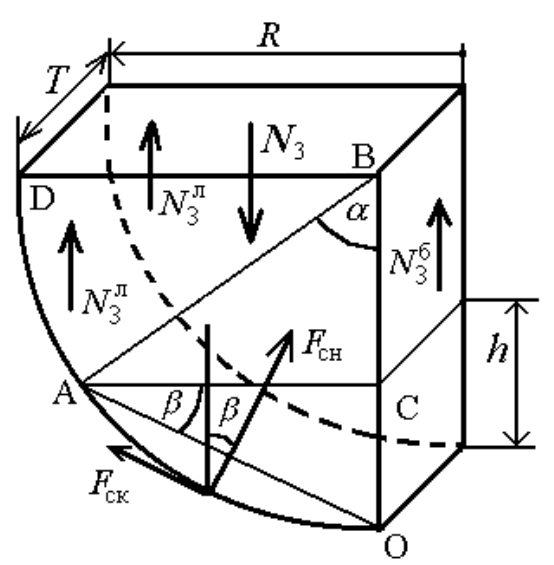

Рис. 4. Лункообразователь секторной формы

Сила $N_{3}$, необходимая для внедрения сектора, в общем виде определится так:

$N_{3}=F_{\mathrm{cH}} \cdot\left(\cos \beta+f_{\mathrm{c}} \cdot \sin \beta\right)+$

$+2 N_{3}^{\pi}+N_{3}^{\sigma}$.

Здесь $F_{\text {сн }}-$ нормальная сила, действующая на криволинейную поверхность сектора; $N_{3}^{\text {л }}$ - касательная сила, действующая по лицевой грани; $N_{3}^{\sigma}$ - касательная сила, действующая по боковой грани. Обозначим $\mathrm{AO}=a$. Тогда величина $F_{\mathrm{cH}}$ получится из уравнения:

$F_{\mathrm{cH}}=a \cdot T \cdot[\sigma]$.

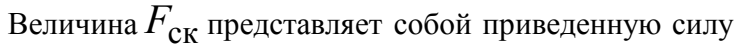
трения, действующую по криволинейной поверхности сектора, и определяется по известной формуле:

$F_{\text {ск }}=F_{\text {сH }} \cdot f_{\mathrm{c}}$.

Выразим тригонометрические значения величин через линейные величины:

$\cos \beta=\frac{\sqrt{2 R h-h^{2}}}{a}, \quad \sin \beta=\frac{h}{a}$.

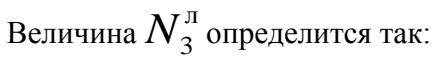

$N_{3}^{\pi}=f_{\mathrm{c}} \cdot[\sigma] \cdot\left[\begin{array}{l}\frac{\pi R^{2}}{360} \arcsin \frac{\sqrt{2 R h-h^{2}}}{R} \\ -\frac{(R-h) \sqrt{2 R h-h^{2}}}{2}\end{array}\right]$.

Величина $N_{3}^{\sigma}$ определится так:

$N_{3}^{\sigma}=T \cdot h \cdot[\sigma] \cdot f_{\mathrm{c}}$.

В развернутом виде выражение (16) выглядит так:

$$
\begin{aligned}
& N_{3}=T \cdot[\sigma] \cdot\left(\sqrt{2 R h-h^{2}}+f_{\mathrm{c}} \cdot h\right)+
\end{aligned}
$$

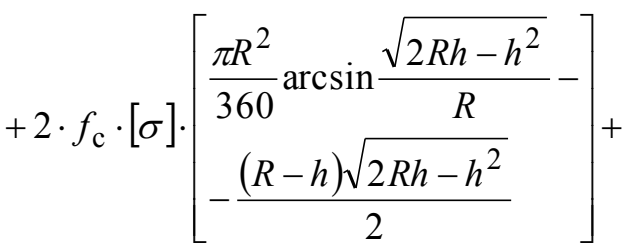

$$
\begin{aligned}
& +T h \cdot[\sigma] \cdot f_{\mathrm{c}} .
\end{aligned}
$$

Максимального значения $N_{3}=N_{3}^{\max }$ сила внедрения достигнет при условии $h=R$.

Подставим эти значения в выражение (21). Получим:

$$
\begin{aligned}
& N_{3}^{\max }=T \cdot[\sigma] \cdot\left(R+f_{\mathrm{c}} \cdot R\right)+ \\
& +\frac{\pi R^{2}}{2} \cdot f_{\mathrm{c}} \cdot[\sigma]+T \cdot R \cdot[\sigma] \cdot f_{\mathrm{c}}= \\
& =[\sigma] \cdot\left[R \cdot T+f_{\mathrm{c}} \cdot\left(2 R \cdot T+\frac{\pi R^{2}}{2}\right)\right] \cdot \\
& \text { Выражение }\left(2 R \cdot T+\frac{\pi R^{2}}{2}\right) \text { в (22) представляет }
\end{aligned}
$$

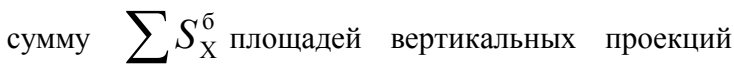
боковых стенок сектора (рис. 4). 
С учетом вышеизложенного представим выражение (22) в следующем виде:

$$
N_{\mathrm{c \Pi}}^{\max }=[\sigma] \cdot\left(S_{\mathrm{B}}+f_{\mathrm{c}} \cdot \sum S_{\mathrm{X}}^{\sigma}\right)
$$

где $S_{\mathrm{B}}$ - площадь верхнего основания сегмента.

\section{СОПРОТИВЛЕНИЕ ВНЕДРЕНИЮ В ПОЧВУ ЛУНКООБРАЗОВАТЕЛЯ ПРОИЗВОЛЬНОЙ ФОРМЫ}

Для выявления общих закономерностей при внедрении в почву деформатора произвольной формы перепишем выражение (11) для лункообразователя конической формы так:

$$
N_{1}=[\sigma] \cdot\left[\pi \cdot R^{2}+\frac{(2 \pi \cdot R+2 \pi \cdot r)}{2} \cdot h \cdot f_{\mathrm{c}}\right] .
$$

В такой записи первое слагаемое в квадратных скобках соответствует площади $S_{\text {в }}$ верхнего основания лункообразователя, первое слагаемое в круглых скобках соответствует периметру $P_{\mathrm{B}}$ верхнего основания, а второе - периметру $P_{\mathrm{H}}$ нижнего основания лункообразователя.

Выражение (7) для лункообразователя в виде четырехугольной пирамиды перепишем так:

$$
N=[\sigma] \cdot\left[T^{2}+\frac{(4 T+4 t)}{2} \cdot h \cdot f_{\mathrm{c}}\right] \text {. }
$$

В такой записи первое слагаемое в квадратных скобках, так же как и в предыдущем примере, представляет собой площадь $S_{\text {в }}$ верхнего основания деформатора (см. рис. 1), первое слагаемое в круглых скобках представляет собой периметр $P_{\mathbf{B}}$ верхнего основания, а второе слагаемое - периметр $P_{\mathrm{H}}$ нижнего основания

Выражение (15) перепишем так:

$N_{2}=[\sigma] \cdot\left(T \cdot L+\frac{2 T+2 L+2 T}{2} h \cdot f_{\mathrm{c}}\right)$.

Здесь:

1. $S_{\mathrm{B}}=T \cdot L$.

2. $P_{\mathrm{B}}=2 T+2 L$.

3. $P_{\mathrm{H}}=2 T$.

Заметим, что последнее не кажется очевидным. Для пояснения представим заостренную нижнюю грань клина как прямоугольник, одна сторона которого равна $T$, а другая имеет малую величину, которой можно пренебречь (принять равной нулю) при определении периметра этого прямоугольника. Буквально следуя определению периметра, запишем:

$P_{\mathrm{H}}=T+0+T+0=2 T$.
Выражение (27) для значения периметра нижней грани клина верно. На основании вышеизложенного выражения (24) - (26) можно представить в универсальном виде:

$N_{\Pi}=[\sigma] \cdot\left(S_{\mathrm{B}}+\frac{P_{\mathrm{B}}+P_{\mathrm{H}}}{2} \cdot h \cdot f_{\mathrm{c}}\right)$,

где $N_{\text {П }}$ - сопротивление внедрению в почву лункообразователей (см. рис. 1-3).

Применимость выражения (28) была проверена и подтверждена расчетами для лункообразователей цилиндрической, пирамидальной трехгранной, пирамидальной четырехгранной с прямоугольным сечением оснований и клиновидной форм.

Таким образом, выражение (27) рекомендуется для определения сопротивления внедрения в почву любых прямолинейных лункообразователей. Сравним его с выражением (23).

Для прямолинейного лункообразователя произведение полусуммы периметров верхнего и нижнего оснований на высоту тождественно сумме площадей вертикальных проекций его боковых стенок.

Отсюда следует, что простое выражение (27), справедливое для прямолинейных лункообразователей, является частным случаем более сложного для расчетов выражения (23), которое можно применять для лункообразователей любой формы.

Пример 2. Определить сопротивление внедрению в почву лункообразователя в виде клина, представленного на рис. 3. Ширина $T$ клина постоянна по высоте и составляет $0,1 \mathrm{м}$; толщина $L$ клина в верхнем сечении 0,04 м; высота $h$ составляет 0,23 м. Характеристика почвы аналогична приведенной в примере 1 .

Решение: Используем выражение (27). Получим:

$N_{\Pi}=280 \cdot 10^{3}$.

$\left(\begin{array}{l}0,1 \cdot 0,04+ \\ +\frac{4 \cdot 0,1+2 \cdot 0,04}{2} \cdot 0,23 \cdot 0,26\end{array}\right)=5139 \mathrm{H}$.

Ответ: $5,14 \mathrm{\kappa H}$.

Пример 3. Определить сопротивление $N_{\text {сп }}^{\max }$ внедрению в почву на полную глубину лункообразователя в виде ступенчатого сектора (рис. 5). Параметры: $R=$ 0,$2 ; T=0,05 ; l=0,02$ м. Характеристика почвы аналогична приведенной в примере 1 . 


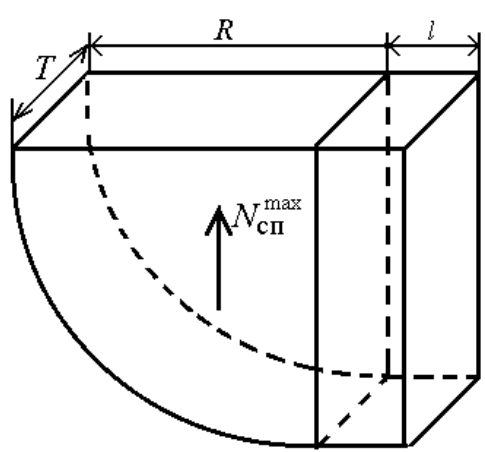

Рис. 5. Лункообразователь в виде ступенчатого сектора

Решение: Используем выражение (23).

$N_{\mathrm{c} \Pi}^{\max }=[\sigma] \cdot\left(S_{\mathrm{B}}+f_{\mathrm{c}} \cdot \sum S_{\mathrm{X}}^{\sigma}\right)=$

$=[\sigma] \cdot\left[T \cdot(R+l)+f \cdot\left(\begin{array}{l}2 \cdot T \cdot R+ \\ +\frac{\pi \cdot R^{2}}{2}+2 \cdot l \cdot R\end{array}\right)\right]$.

$N_{\mathrm{c} \Pi}^{\max }=280 \cdot 10^{3}$.

$\cdot\left[\begin{array}{l}0,05 \cdot(0,2+0,02)+ \\ +0,26 \cdot\left(\begin{array}{l}2 \cdot 0,05 \cdot 0,2+\frac{3,14 \cdot 0,2^{2}}{2} \\ +2 \cdot 0,02 \cdot 0,2\end{array}\right)\end{array}\right]=$

$=9690,24 \mathrm{H}$.

Ответ: 9,7 кН.

\section{ЭНЕРГИЯ ВНЕДРЕНИЯ} ЛУНКООБРАЗОВАТЕЛЯ

Рассмотрим случаи расчета энергии внедрения для наиболее часто применяемых распространенных лункообразователей конической и клиновидной формы (см. рис. 2 и 3 ).

Сила сопротивления внедрению лункообразователя по мере заглубления в почву является переменной величиной, обозначим ее текущее значение $N_{x}$. Обозначим величиной $x$ текущее значение заглубления, которое изменяется от 0 до $h$.

Величина $W$ энергии, затрачиваемой на преодоление силы $N_{x}$ при перемещении на некоторое расстояние $h$, определяется по известной формуле:

$W=\int_{0}^{h} N_{x} \mathrm{~d} x$

\section{Энергия внедрения лункообразователя клиновид-}

\section{ной формы}

Определим энергию внедрения $W_{2}$ для деформатора клиновидной формы (см. рис. 3).

Для определения текущего значения силы $N_{x 2}$ воспользуемся выражением (15), представив текущие величины толщины $L_{x}$ клина на уровне пересечения с почвой и заглубления $h$ через $x$ :

$L_{x}=2 x \cdot \operatorname{tg} \alpha ; \quad h=x$. Тогда:

$N_{x 2}=[\sigma] \cdot\left(\begin{array}{l}2 T \cdot x \cdot f_{\mathrm{c}}+2 x^{2} \cdot \operatorname{tg} \alpha \cdot f_{\mathrm{c}}+ \\ +2 T \cdot x \cdot \operatorname{tg} \alpha\end{array}\right)$.

Подставляя (30) в (29) и решая определенный интеграл, получим:

$W_{2}=[\sigma] \cdot\left(\begin{array}{l}T \cdot h^{2} \cdot f_{\mathrm{c}}+\frac{2}{3} \cdot h^{3} \cdot \operatorname{tg} \alpha \cdot f_{\mathrm{c}}+ \\ +T \cdot h^{2} \cdot \operatorname{tg} \alpha\end{array}\right)$.

Выразив $\operatorname{tg} \alpha$ через линейные величины (см. (14) (15)), получим в окончательном виде:

$$
W_{2}=[\sigma] \cdot\left(\begin{array}{l}
T \cdot h^{2} \cdot f_{\mathrm{c}}+\frac{1}{3} \cdot L \cdot h^{2} \cdot f_{\mathrm{c}}+ \\
+\frac{1}{2} \cdot T \cdot L \cdot h
\end{array}\right) .
$$

Пример 4. Определить энергию внедрения в почву лункообразователя в виде клина, представленного на рис. 3. Ширина $T$ клина постоянна по высоте и составляет 0,1 м; толщина $L$ в верхнем сечении 0,04 м; высота $h$ составляет 0,23 м. Характеристика почвы аналогична приведенной в примере 1 .

Решение: Используем выражение (31):

$W_{2}=280 \cdot 10^{3} \cdot\left(\begin{array}{l}0,1 \cdot 0,23^{2} \cdot 0,26+ \\ +\frac{1}{3} \cdot 0,04 \cdot 0,23^{2} \cdot 0,26+ \\ +\frac{1}{2} \cdot 0,1 \cdot 0,04 \cdot 0,23\end{array}\right)=$

$=565,26$ Дж.

Ответ: 0,57 кДж.

Энергия внедрения лункообразователя конической формы

Для определения текущего значения силы $N_{x 1}$ воспользуемся выражением (10).

$R_{x}=r+x \cdot \operatorname{tg} \alpha ; \quad h=x$. 


$$
\begin{aligned}
& N_{x 1}=\pi \cdot[\sigma] . \\
& \cdot\left[\begin{array}{l}
r^{2}+x \cdot\left(2 r \cdot \operatorname{tg} \alpha+2 r \cdot f_{\mathrm{c}}\right)+ \\
+x^{2} \cdot\left(\operatorname{tg}^{2} \alpha+\operatorname{tg} \alpha \cdot f_{\mathrm{c}}\right)
\end{array}\right] .
\end{aligned}
$$

Подставляя (32) в (29) и решая определенный интеграл, получим:

$$
\begin{aligned}
& W_{1}=\pi \cdot[\sigma] . \\
& {\left[\begin{array}{l}
x \cdot r^{2}+x^{2} \cdot r \cdot\left(\operatorname{tg} \alpha+f_{\mathrm{c}}\right)+ \\
+x^{3} \cdot \frac{\operatorname{tg}^{2} \alpha+\operatorname{tg} \alpha \cdot f_{\mathrm{c}}}{3}
\end{array}\right] .}
\end{aligned}
$$

Пример 5. Определить сопротивление и энергию внедрения в почву лункообразователя в виде полного конуса с радиусом основания 0,1 м и высотой 0,2 м. Характеристика почвы аналогична приведенной в примере 1 .

Решение: Используем выражения (11) и (33), упростив их с учетом того, что для полного конуса радиус малого основания $r=0$.

В этом случае: $\operatorname{tg} \alpha=\frac{R}{h}$.

Сопротивление внедрению определится так:

$N_{1}=\pi \cdot[\sigma] \cdot\left(R^{2}+f_{\mathrm{c}} \cdot h \cdot R\right)=$

$=3,14 \cdot 280000 \cdot\left(0,1^{2}+0,26 \cdot 0,2 \cdot 0,1\right)=$

$=13363,84 \mathrm{H}$.

Энергия внедрения определится так:

$W_{1}=\pi \cdot[\sigma] \cdot \frac{h^{3}}{3} \cdot\left(\frac{R^{2}}{h^{2}}+\frac{R}{h} \cdot f_{\mathrm{c}}\right)=$

$=3,14 \cdot 280000 \cdot \frac{0,2^{3}}{3} \cdot\left(\frac{0,1^{2}}{0,2^{2}}+\frac{0,1}{0,2} \cdot 0,26\right)=$

$=890,92$ Дж .

Ответ: 13,4 кН; 0,9 кДж.
Предлагаемая методика для ее реализации требует знания элементарных механических характеристик почвы: сопротивления почвы смятию $[\sigma]$ и коэффициента трения сталь-почва $f_{\mathrm{c}}$, которые могут быть определены опытным путем.

Данные о почве для использования методики в приближенных и учебных расчетах приведены в табл. 1 .

Таблица 1

Механические характеристики почвы

\begin{tabular}{|l|c|c|}
\hline $\begin{array}{l}\text { Механический состав } \\
\text { почвы и условия работ }\end{array}$ & {$[\sigma]$} & $f_{\mathrm{c}}$ \\
\cline { 2 - 3 } & кПа & - \\
\hline $\begin{array}{l}\text { Минеральная на } \\
\text { вырубках }\end{array}$ & 1370 & 0,25 \\
\hline $\begin{array}{l}\text { Гумусная на } \\
\text { вырубках }\end{array}$ & 280 & 0,27 \\
\hline $\begin{array}{l}\text { Супесчаная бедная } \\
\text { в питомниках }\end{array}$ & 108 & 0,25 \\
\hline $\begin{array}{l}\text { Супесчаная гумусная в } \\
\text { питомниках }\end{array}$ & 86 & 0,30 \\
\hline $\begin{array}{l}\text { Суглинистая } \\
\text { старопахотная }\end{array}$ & 65 & 0,40 \\
\hline $\begin{array}{l}\text { Суглинистая } \\
\text { целинная }\end{array}$ & 450 & 0,30 \\
\hline
\end{tabular}

